\title{
SARS-CoV-2 infection in a child with Fanconi anemia and determined immunosuppressed status
}

\author{
Claudia Chirila', Geta Vancea ${ }^{1,2}$, Dana Ispas', Nicoleta Voicu-Parvu', Nicoleta Tudor', \\ Gabriela Scurtu', Andreea Florentina Stoenescu ${ }^{1,2}$, Andreea Popica', Raluca Popescu', \\ Emanoil Ceausu ${ }^{1,2,3}$, Simin-Aysel Florescu ${ }^{1,2}$ \\ 1 "Dr. Victor Babes" Clinical Hospital for Infectious and Tropical Diseases, Bucharest, Romania \\ 2 "Carol Davila" University of Medicine and Pharmacy, Bucharest, Romania \\ ${ }^{3}$ Academy of Medical Sciences, Romania
}

\begin{abstract}
Introduction. SARS-CoV-2 virus infection affects all age groups. In children, the infection mainly causes asymptomatic or mildly symptomatic forms of the disease, regardless of their immune status.

Case presentation. We describe the case of a 7-year-old male child, known to have Fanconi anemia, scheduled for bone marrow transplantation. The patient comes from a family outbreak of COVID-19, which is why he was tested for SARS-CoV-2 infection. He is asymptomatic at the time of admission to our clinic. The clinical examination performed at the time of admission shows a patient in good general condition, afebrile, with pale skin and mucous membranes, without respiratory changes. Paraclinically, severe neutropenia, severe normochromic normocytic anemia and severe thrombocytopenia are detected, for which transfusions of erythrocyte mass and platelet mass are performed. Due to the immunocompromised status, antibiotic therapy is instituted. If necessary, symptomatic treatment is administered. The evolution is favorable, and the SARS-CoV-2 RT-PCR control test is negative on the eighth day of hospitalization.

Conclusions. Immunocompromised status is not a major risk factor for severe COVID-19 in children.
\end{abstract}

ABSTRACT

Keywords: SARS-CoV-2, COVID-19, Fanconi anemia, child, immunocompromised

\section{INTRODUCTION}

SARS-CoV-2 is a new coronavirus first detected in December 2019 in the Wuhan region of China. Subsequently, the infection with this virus (COVID-19) became a global problem, with the World Health Organization declaring a state of pandemic on March 11, 2020. COVID-19 affects all age groups, children generally developing asymptomatic or mildly symptomatic forms, compared to adults who develop more frequently moderate or severe forms (1-4). In children, immunocompromised status, without the association of other comorbidities, is not a major risk factor for the development of severe forms of the disease. To date, there are several theories that try to explain this evolution, including: structural and functional differences in the immune system of children compared to adults, the concomitant presence of several viruses in the nasopharynx in children with potential competition between them and variable secondary viral load, the difference in expression of ACE receptors underlying the pathogenesis of COVID-19. Despite the large number of theories, there is currently little concrete data to explain this. In most cases, children 
do not need specific treatment for SARS-CoV-2 infection $(5,6)$.

\section{CASE PRESENTATION}

\section{Presenting concerns}

We describe the case of a 7-year-old male child, who is hospitalized between August $10^{\text {th }} 2020-\mathrm{Au}-$ gust $18^{\text {th }} 2020$ in the Clinical Hospital for Infectious and Tropical Diseases "Dr Victor Babeș” Bucharest by transfer from Giurgiu County Hospital, where he was tested for SARS-CoV-2 infection because 3 members of his family were previously diagnosed with this infection. The patient was asymptomatic both at the time of admission in Giurgiu County Hospital and at the time of admission in our clinic. We note from the patient's personal medical history that he was diagnosed with Fanconi anemia in October 2019. The patient has a chemotherapy catheter mounted on the superior vena cava, but the mother cannot specify the child's treatment schedule and has no medical evidence. On August $12^{\text {th }} 2020$ the patient was scheduled for bone marrow transplant. The informed consent of the mother was obtained in order to carry out both the child's clinical examination, paraclinical investigations and treatment.

\section{Clinical findings}

Objective examination at admission reveals pale skin and mucous membranes, with no visible active bleeding and no other changes.

\section{Diagnostic focus and assessment}

Paraclinical investigations performed at the time of admission are complete blood count, markers of inflammation and sepsis (C-reactive protein, ESR, procalcitonin), coagulation tests, AST, ALT, LDH, urea, creatinine, blood sugar, anti-HIV antibodies, ionogram, nasal exudate, pharyngeal exudate, urinalysis, urine culture, blood group and Rh screening, Coombs test, pulmonary radiography. These reveal significant leukopenia $(2,800 / \mu \mathrm{l})$ with severe neutropenia $(300 / \mu \mathrm{l})$, severe normochromic normocytic anemia $(\mathrm{Hb}=7.3 \mathrm{~g} / \mathrm{dl})$ and severe thrombocytopenia $(15,000 / \mu \mathrm{l})$. Regarding the inflammatory syndrome, there are discreetly increased C-reactive protein $(0.48 \mathrm{mg} / \mathrm{dl})$, ESR and procalcitonin within normal limits. Ddimers have a slightly increased value $(0.8 \mu \mathrm{g} / \mathrm{ml})$, $\mathrm{LDH}$ is discreetly increased (308 UI/l) and minimal hepatic cytolysis syndrome is detected (ALT $=80 \mathrm{U} / \mathrm{l})$. The pulmonary radiography performed reveals a moderate accentuation of the bilateral peribronhovascular interstitial pattern.

\section{Therapeutic focus and assessment}

Considering the associated pathology manifested by severe anemia and severe thrombocytopenia, $200 \mathrm{ml}$ of erythrocyte mass and $280 \mathrm{ml}$ of irradiated platelet mass isogroup isoRh were administrated on August $12^{\text {th }}$ 2020. The transfusions were performed without incidents, the complete blood count performed on August $13^{\text {th }}$ revealing $\mathrm{Hb}$ with the value of $10 \mathrm{~g} / \mathrm{dl}$ and the improvement of thrombocytopenia up to $121,000 / \mu l$. It is decided to institute antibiotic therapy with Ceftriaxone $2 \mathrm{~g} /$ day with administration in 2 doses/day, at an interval of 12 hours, for 7 days. Oral Paracetamol has also been recommended in case of fever or pain.

On the eighth day of hospitalization, the patient is retested by RT-PCR SARS-CoV-2 test, according to the national protocol, and the result is negative. The mother requests discharge on request, before the 10-day hospitalization period provided in the protocol.

During hospitalization, the patient complained of a rare dry cough, which recovered quickly. At discharge, the general condition was good, the patient was afebrile, with pale skin and mucous membranes, with bilateral vesicular murmur present, no added rales, no cough, with $\mathrm{SaO} 2=98 \%$ in aa, rhythmic heart sounds, $100 \mathrm{bpm}$, without symptoms or signs concerning the digestive system, with present diuresis.

\section{Follow up and monitoring}

At discharge, it was recommended to monitor health status through the family doctor, according to the national protocol.

\section{DISCUSSIONS}

In the pediatric population, SARS-CoV-2 infection mostly causes asymptomatic or mildly symptomatic forms of the disease. The most common symptoms are fever and cough. Dyspnea, rhinorrhea, sore throat, headache, asthenia, gastrointestinal manifestations such as diarrhea, vomiting or abdominal pain may also occur $(1,3)$.

SARS-CoV-2 infection progresses in three phases: the viral load phase, the lung damage phase and the hyperimmune phase, the latter being associated with an increased risk of mortality (7).

Age is a major protective factor in viral infections in children, a category that also includes COVID-19. Several theories have been issued that explain the favorable evolution within this category of population. There are fewer ACE2 receptors in children's nasal epithelium, so the virus has fewer ways to enter the body. The immune system of children is different from that of adults functionally, in the way that there is less chance that, in the context of a 
pediatric viral infection, the immune processes carried out will cause an excessive inflammatory response. Underlying this functioning are the reduced capacity to up-regulate ACE2 receptors following excessive interferon synthesis, better controlled viral replication by interferon, moderate macrophage activation with consequently low cytokine synthesis. Immunocompromised status is an additional protective factor, which minimizes the chances of progression to the hyperimmune phase $(6,8)$.

This case presents a child with immunocompromised status in the context of Fanconi anemia, which comes from the family outbreak of COVID-19. The peculiarity of the case is that, despite leukopenia with severe neutropenia, severe anemia and severe thrombocytopenia, the patient was mildly symptomatic during the course of the disease, with minimal paraclinical changes due to SARS-CoV-2 infection and mostly due to its underlying disease, unlike adult patients with immunosuppressed status, who develop moderate to severe forms of COVID-19. The child did not require specific treatment for
SARS-CoV-2 infection, but antibiotic therapy with Ceftriaxone was instituted for prophylactic purposes, given his immunosuppressed status.

We consider that, in the case of our patient, both his age and his immunocompromised status contributed to the development of this mildy symptomatic form, without reaching the hyperimmune phase.

\section{CONCLUSIONS}

Following infection with the SARS-CoV-2 virus, the child developed a form of mildly symptomatic disease. Paraclinically, minimal changes were detected due to SARS-CoV-2 infection, most of them were caused by the underlying disease. Fanconi's anemia, due to the immunodepressed status it causes, did not contribute to the appearance of a severe form of the disease. The patient's evolution was rapidly favorable, without specific treatment for SARSCoV-2 infection.

Conflict of interest: none declared Financial support: none declared

\section{REFERENCES}

1. Deville JG, Song E, Ouellette CP. Coronavirus disease 2019 (COVID-19): Clinical manifestations and diagnosis in children. UpToDate, 2020.

2. Yunus Akçabelen, Ayça Koca Yozgat, Aslinur Ozkaya-Parlakay, et al. COVID-19 in a child with severe aplastic anemia. Available at: https:// www.authorea.com/users/320136/articles/449722-covid-19-in-achild-with-severe-aplastic-anemia.

3. Rathore V, Galhotra A, Pal R, Sahu KK. COVID-19 Pandemic and Children: A Review. J Pediatr Pharmacol Ther. 2020;25(7):574-585.

4. Coronavirus WorldWide Graphs. Available at: https://www. worldometers.info/coronavirus/worldwide-graphs.

5. Minotti C, Tirelli F, Barbieri E, Giaquinto $C$, Donà D. How is immunosuppressive status affecting children and adults in SARS-CoV-2 infection? A systematic review. J Infect. 2020 Jul;81(1):e61-e66.

6. Brodin P. Why is COVID-19 so mild in children? Acta Paediatr. 2020 Jun;109(6):1082-1083.

7. Voicu V, Cernescu C, Popescu I. Pandemia COVID-19 in România: aspecte clinice și epidemiologice. București: Editura Academiei Române, 2020:147.

8. Nicastro E, Verdoni L, Bettini LR, Zuin G, Balduzzi A, Montini G, Biondi A, D'Antiga L. COVID-19 in Immunosuppressed Children. Front Pediatr. 2021 Apr 29;9:629240. 